\title{
Use of Stereology to Derive a New Kinetic Equation for Mean Curvature Driven Grain Growth
}

\author{
Robert T. DeHoff, Burton R. Patterson, Catherine A. Sahi, Steven Chiu \\ Department of Materials Science and Engineering \\ University of Florida \\ Gainesville, FL 32611
}

Key words: grain growth; Monte Carlo simulation; three dimensions; kinetics; stereology

\begin{abstract}
The idea that normal grain growth driven by surface tension may be described theoretically by the hypothesis that the local velocity of an element of grain boundary is proportional to its local mean curvature dates back more than half a century. Von Neumann was the first to derive this relation and used it to predict the rate of evolution of a two dimensional cell structure. MacPherson and Srolovitz extended this development to describe growth in three dimensions; however, their result was couched in terms that did not facilitate tests of the theory. In this paper expected value theorems established in stereology are invoked to extend their result to provide a new equation that predicts the rate of change of volume of grains in a microstructure which, while preserving the rigor and generality of the result, expresses it in terms of quantities that can be measured in microstructures. This is illustrated with a set of measurements based upon the theory derived from a grain growth simulation that successfully tests its predictions. It is interesting that this result also exhibits an " $n-6$ rule" that is similar to, but not identical with, that contained in von Neumann's theory.

(C) 2015. This manuscript version is made available under the Elsevier user license http://www.elsevier.com/open-access/userlicense/1.0/
\end{abstract}




\section{Introduction}

In 1951 [1] von Neumann presented what has become a classical theory of coarsening of a two dimensional soap film network at a conference on grain growth as a written discussion to a paper by Cyril Stanley Smith [2]. This theory applies Gibbs' description of the thermodynamics of systems with curved interfaces [3] to derive the result that the local velocity of an element of soap film in this coarsening process is proportional to its local curvature. The theory assumes that the motion is controlled by the rate of diffusion of atoms of the gas filling the bubbles on both sides through the film. His theory also predicts the remarkable result that the rate of growth of the area of a given soap cell is completely determined by the number of corners it has, a topological property of the cell, and is independent of the details of the geometry of the cell boundaries. He showed that cells with less than six corners shrink while those with more than six corners grow. This classical result has become known as the " $n$ - 6 rule"; it has fascinated researchers for more than half a century.

As a consequence researchers in grain growth and related areas have sought to extend von Neumann's theory to three dimensional cell structures. Most successful of these developments is that by MacPherson and Srolovitz [4], which starts with the analogous three dimensional version of von Neumann's prediction, i.e., the local velocity of an element of surface is proportional to its local mean curvature, and derives an equation for the rate of change of the volume of each cell in the three dimensional system:

$$
\frac{d V}{d t}=-2 \pi M \gamma\left(L(D)-\frac{1}{6} \sum_{i=1}^{n} e_{i}(D)\right)
$$


where $\mathcal{M}$ is a mobility, $\gamma$ is the surface tension, $\mathcal{L}(\mathrm{D})$ is a "natural measure of the linear size" of the polyhedral cell, an abstruse geometric property carefully defined in their text, and $e_{i}$ is the length of an edge of the polyhedron and the summation is carried over the set of edges. This rigorous three dimensional derivation provides the definitive solution to the quest for a three dimensional version of von Neumann's fascinating result.

This mathematically rigorous but abstract result does not provide a pathway for designing tests of the theory, either in the physical world or in simulations. To this end this paper presents a derivation of the three dimensional version of the von Neumann equation that yields a new final result (an extension of MacPherson and Srolovitz' equation) that is also without geometric assumptions and is expressed in terms of stereological parameters that can be evaluated in simulations or serial sectioning experiments. This new result has a tantalizing doff of the cap to von Neumann's two dimensional equation involving his topological parameter, the number of corners per grain. However it is demonstrated that other metric parameters are also involved in determining the rate of volume change of a grain, and the result is thus not topological as von Neumann showed in two dimensions. This presentation focuses on the new version of the theory, and an assessment of its predictions using our grain growth simulation.

\section{Kinetic Theory of Grain Growth}

The classical phenomenological hypothesis in the theory of Mullins and others [5-7] of surface tension driven grain growth suggests that an element of grain boundary area moves toward its center of curvature with a local velocity, $\mathrm{v}$, which is proportional to the local mean curvature of that element, $\mathrm{H}$ : 
where

$$
H=\frac{1}{2}\left(\frac{1}{r_{1}}+\frac{1}{r_{2}}\right)
$$

Here $r_{1}$ and $r_{2}$ are the local principal radii of curvature of the surface element. The conventions chosen here are defined with respect to any isolated grain. Focus on an element of area of the faces of the grain. The local velocity associated with an element of surface is defined to be positive if the element moves in the outward direction thus increasing the volume of the grain; $v$ is negative if directed inward, contributing a decrease in the volume of the grain. Similarly the sign of each local radius of curvatures is positive if its curvature vector points toward the inside and negative if outside. Convex surface elements result when both radii are positive ( $\mathrm{H}$ is positive), saddle surface elements occur when they have opposite signs ( $\mathrm{H}$ may be positive or negative, depending upon the values of $r_{1}$ and $r_{2}$ ), while concave elements are characterized by both curvatures being negative $(\mathrm{H}$ is negative). Accordingly, convex surface elements move inward $(v$ is negative), concave elements move outward ( $v$ is positive), and saddle elements may move inward or outward depending upon the values of $r_{1}$ and $r_{2}$. Small grains are bounded by mostly convex surface elements and shrink and disappear; surface elements of very large grains are mostly concave and grow.

The coefficient $\mathrm{k}$ is a kinetic factor containing the grain boundary energy and mobility of the boundary element. 
Von Neumann derived his kinetic equation in his two dimensional example based upon the assumption that the boundary migration is controlled by the diffusion of gas atoms through the film with boundary conditions at the two interfaces bounding the film determined by local equilibrium with the curved surfaces. Since these boundary elements are curved, local equilibrium concentrations are determined in part by the local curvature of these surfaces, the capillary shift. This curvature dependence carries through in the evaluation of the diffusion fluxes in the liquid and thence to the velocity of the moving interfaces. This scenario does not describe the grain growth process. In grain growth, there is no film separating adjacent grains: only one interface; no capillarity shifted interface concentrations determining a rate of volume diffusion; indeed there are no volume diffusion fluxes that determine the local velocity of the boundary interfaces. The inclusion of the local curvature comes not from an assumption of a capillarity shifted local interfacial equilibrium, but from a comparison of the thermodynamics at a curved interface with that at an analogous flat one. In its application to grain growth Eq. (2) is not a derived kinetic equation; indeed it has the status of a plausible phenomenological hypothesis.

The rate of change of the volume of any given grain may be computed from the distribution of local velocities, $\mathrm{v}$, over its faces by invoking the kinematic equation [8]:

$$
\frac{d V}{d t}=\iint_{S} v d S=\iint_{S}-k H d S=-k \iint_{S} H d S=-k M_{S}
$$

where the integration is carried over the areas $S$ of the surfaces of the grain faces; $M_{S}$ is called the integral mean curvature of the faces, and thus becomes the central geometric factor in the 
analysis:

$$
M_{S}=\iint_{S} H d S
$$

The kinetic factor, $\mathrm{k}$, is assumed to be constant in this theory. Relaxation of this assumption can lead to generalizations of the theory, but is beyond the scope of this paper.

The remainder of the development focuses upon the evaluation of $\mathrm{M}_{\mathrm{S}}$ in terms of measurable quantities.

The integral mean curvature $\mathrm{M}$ of a polyhedron, see Appendix A, such as a single grain, with curved edges and faces has contributions from both its faces $(\mathrm{S})$ and edges $(\mathrm{E})[9,10]$ :

$$
M=M_{S}+M_{E}=M_{S}+\frac{1}{2} \int_{L} \chi d L
$$

where $\mathrm{L}$ is length of edges on the polyhedron and $\chi$ is the local dihedral angle, i.e., angle between the surface normals to the elements of cell faces that meet to form an element of edge. From Eq. (5) the geometric parameter at the center of interest in this kinetic theory of grain growth is

$$
M_{S}=M-\frac{1}{2} \int_{L} \chi d L
$$

or 


$$
M_{S}=M-\frac{1}{2} \frac{\int_{L} X d L}{\int_{L} d L} \int_{L} d L=M-\frac{1}{2}<\chi>L
$$

where $\langle\chi\rangle$ is here defined to be the average dihedral angle along the edges of the polyhedron.

The kinetic equation for any given grain in the structure may be obtained by inserting Eq. (7) into Eq. (4):

$$
\frac{d V}{d t}=-k\left(M-\frac{1}{2}<\chi>L\right)
$$

This equation applies separately and without any simplifying geometric assumptions to every grain in the structure. Comparison with MacPherson and Srolovitz, Eq. (1), shows that

$$
M=2 \pi L(D) \quad \text { and } \quad \frac{1}{2}<\chi>L=\frac{\pi}{3} \sum_{i=1}^{n} e_{i}(D)
$$

in their notation.

\section{Stereological Relationships}

The geometric properties in Eq. (8) can be evaluated conceptually and experimentally without assumption by applying two of the fundamental relationships of stereology [11]. The stereological equations for these parameters are generally formulated in terms of values per unit volume of microstructure. Thus $\mathrm{M}_{\mathrm{V}}$ is the integral mean curvature of a set of polyhedral features per unit volume, $\mathrm{M}_{\mathrm{VS}}$ is the integral mean curvature of their faces per unit volume, and $\mathrm{L}_{\mathrm{V}}$ is the total length of their edges per unit volume. 
The pertinent stereological equations are [12-14]:

$$
M_{V}=2 \pi N_{A}
$$

and

$$
L_{V}=2 P_{A}
$$

where $\mathrm{N}_{\mathrm{A}}$ is the expected value of the number of feature sections (in this application, the grain sections highlighted in Figure 1) per unit area of a stereological sampling plane through the structure and $\mathrm{P}_{\mathrm{A}}$ is the expected value of the number of points of emergence per unit area of lineal features (grain edges in this case, marked with white circles in Figure 1, - the number of grain corners) on that plane. These relationships contain no simplifying assumptions about the geometries of the features being sampled; specifically, Eq. (10) is not limited to convex features $[12,13]^{1}$.They are among the fundamental expected value theorems of stereology which require that measurements be made on sampling planes selected uniformly and isotropically from the population of planes in three dimensional space. Indeed, this requirement forms the basis for the design of sampling schemes in stereology [15]. If the structure is spatially uniform and isotropic,

\footnotetext{
${ }^{1}$ Departures from convexity are manifest as multiple sections through the same particle and/or feature sections with holes in them. In this general case, $N_{A}=N_{A+}-N_{A-}$, where $\mathrm{N}_{\mathrm{A}+}$ is the total number of section boundaries that enclose a grain (including extra ones that arise from

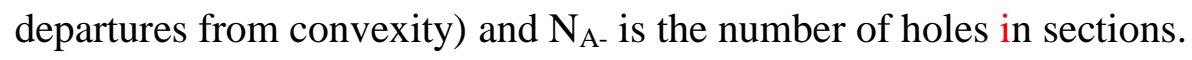


as is generally true in simple grain growth, any random sampling plane satisfies these requirements.

\section{Growth Rate Equation for an Individual Grain}

The growth rate Eq. (8) can be expressed in terms of measurable quantities on sections through that grain through the following development. It is made circuitous by the need to express $\mathrm{M}$ and L in Eq. (8) in the form of the stereological Eqs. (10) and (11) formulated as they are as results for global properties per unit volume. To obtain the result for a single grain, imagine a structure consisting of a large number per unit volume $\mathrm{N}_{\mathrm{V}}$ of grains identical to that grain, distributed uniformly and isotropically in a volume. The integral mean curvature per unit volume of the surfaces of this collection of grains in this structure is, from Eq. (7),

$$
M_{V S}=N_{V} M_{S}=N_{V} M-\frac{1}{2}<\chi>N_{V} L=M_{V}-\frac{1}{2}<\chi>L_{V}
$$

where $M_{V}$ is the integral mean curvature of the set of grains per unit volume and $L_{V}$ is the length per unit volume of their edges. Apply the stereological Eqs. (10) and (11) to this structure:

$$
M_{V S}=2 \pi N_{A}-\frac{1}{2}<\chi>2 P_{A}
$$

Equation. (13) is the basis for the experimental determination of $\mathrm{M}_{\mathrm{VS}}$ in microstructures. Factor out $2 \pi \mathrm{N}_{\mathrm{A}}$ :

$$
M_{V S}=2 \pi N_{A}\left(1-\frac{1}{2}<\chi>\frac{2 P_{A}}{2 \pi N_{A}}\right)
$$


Recognize that $\mathrm{P}_{\mathrm{A}} / \mathrm{N}_{\mathrm{A}}$ is the ratio of the total number of grain corners (points of emergence of grain edges) on sections through the grains to the total number of grain sections, i.e.,

$$
\frac{p_{A}}{N_{A}}=<c>
$$

$<c>$ is the number of corners per grain on a representative stereological section through the structure. In any general three dimensional cell structure with triple lines, the average dihedral angle at the edges can be shown to be

$$
<\chi>=\frac{\pi}{3}
$$

In a surface tension controlled three dimensional cell structure the dihedral angle for the three grains incident on a triple line are equal and therefore are each $\pi / 3$ at every point on all edges. Assume that this average applies to each grain in the structure. Then

$$
M_{V S}=M_{V}\left(1-\frac{1}{2 \pi} \frac{\pi}{3}<c>\right)=M_{V}\left(1-\frac{1}{6}<c>\right)
$$

This is the result for this collection of $\mathrm{N}_{\mathrm{V}}$ identical grains; the value for one grain in this structure is

$$
M_{S}=M_{V S} / N_{V}=M_{V} / N_{V}(1-1 / 6<c>)=M\left(1-1 / 6<c \geq M\left(\frac{6-<c>}{6}\right)\right.
$$


Alternatively, factor out $\mathrm{P}_{\mathrm{A}}$ in Eq. (13):

$$
\begin{gathered}
M_{V S}=2 P_{A}\left(\frac{2 \pi N_{A}}{2 P_{A}}-\frac{1}{2}\langle\chi>)\right. \\
M_{V S}=L_{V}\left(\pi \frac{N_{A}}{P_{A}}-\frac{1}{2} \frac{\pi}{3}\right)=\pi L_{V}\left(\frac{1}{<0\rangle}-\frac{1}{6}\right)
\end{gathered}
$$

Divide both sides by $\mathrm{N}_{\mathrm{V}}$ :

$$
M_{S}=\frac{M_{V S}}{N_{V}}=\pi \frac{L_{V}}{N_{V}}\left(\frac{1}{<0\rangle}-\frac{1}{6}\right)=\pi L\left(\frac{6-\ll 0\rangle}{6<0\rangle}\right)
$$

Insert either Eq. (18) or (20) into the kinetic Eq. (4):

$$
\frac{d V}{d t}=-k M_{S}=-k M\left(\frac{6-\langle 0\rangle}{6}\right)=-k \pi L\left(\frac{6-\langle 0\rangle}{6<0\rangle}\right)
$$

This result shows that the sign of the volume growth rate for grains is determined by the stereologically averaged number of corners on a plane section: when $\langle c\rangle>6$, the rate of growth is positive; for $\langle\mathrm{c}\rangle\langle 6$ the rate is negative and the condition for zero growth rate of a cell is $\langle\mathrm{c}\rangle=$ 6. The derivation of this result requires three assumptions: the initial kinetic hypothesis, Eq. (2); that the kinetic factor $\mathrm{k}$ is constant in the system; and that $\langle\chi\rangle$ is $\pi / 3$ for the set of grains in each size class. No additional simplifications are required. 
The central quantity at this point, $\langle c\rangle$, can be visualized conceptually, but it is useful to illustrate some typical values of this property of a polyhedron. The calculation of $\langle c\rangle$ for some typical grain shapes, i.e., polyhedral shapes with curved faces and edges, would require computer modeling. However the computation can be carried out analytically for flat faced polyhedra as shown in Appendix B. Results for some regular flat faced polyhedra with from four (a tetrahedron) through fourteen faces (the Kelvin Tetrakaidecahedron) are shown in Table 1.

Table 1. Values of $\langle\mathrm{c}>$ for selected regular polyhedra.

\begin{tabular}{||l|c|c|c||}
\hline \multicolumn{1}{|c|}{ Polyhedron } & Faces & $\langle\chi\rangle$ & $<\mathbf{c}\rangle$ \\
\hline \hline Tetrahedron & 4 & $\cos ^{-1}(-1 / 3)=1.91063$ & 3.288 \\
\hline Triangular Prism & 5 & {$[6(\pi / 2)+2(2 \pi / 3)] / 9=(5 / 9) \pi$} & 3.600 \\
\hline Cube & 6 & $\pi / 2$ & 4.000 \\
\hline Octahedron & 8 & $\cos ^{-1}(1 / 3)=1.2309$ & 5.104 \\
\hline Tetrakaidecahedron & 14 & {$\left[24 \cos ^{-1}(1 / \sqrt{3})+12 \cos ^{-1}(1 / 3)\right] / 36=\pi / 3$} & 6.000 \\
\hline
\end{tabular}

It is noteworthy that the value for $\langle c\rangle$ for the tetrakaidecahedron is 6.000. Kelvin [16] proposed this shape as a model for a stable space-filling cell structure with triple lines and quadruple points. According to the kinetic Eq. (21) this structure has zero growth rate, i.e., is stable.

\section{Test of the Monte Carlo Potts Model Against this Hypothesis}


The kinetic hypothesis, Eq. (2) yields the kinetic prediction in Eq. (21). This prediction applies separately to every grain in the structure at every instant in time. It has been used in a test of an application of a Monte Carlo Potts Model computer simulation as part of a more comprehensive study of grain growth. Among other things, this simulation tracks the volume of every grain in the structure as a function of time. To test the hypothesis the range of volumes is divided into size classes, with volumes in the range $V_{i}$ to $V_{i}+\Delta V_{i}$ defining the $i^{\text {th }}$ size class. A version of the kinetic equation that applies to the subset of grains in a given size class is derived below and applied to simulation information for each class.

Focus upon the subset of grains in the structure with volumes in size class i. Equation (8) applies separately to each grain in this subset. Next focus on the $\mathrm{j}^{\text {th }}$ grain in this subset; designate the geometric properties of this grain as $\mathrm{V}_{\mathrm{j} i}, \mathrm{M}_{\mathrm{ji}}$ and $\mathrm{L}_{\mathrm{ji}}$. Eq. (8) applies to this grain with the specific notation:

$$
\frac{d V_{j i}}{d t}=-k\left(M_{j i}-\frac{1}{2}<\chi_{j i}>L_{j i}\right)
$$

An analogous relation applies to each grain in size class i. Let $V_{i}{ }^{T}$ be the total volume of grains in size class $i$, where

$$
V_{i}^{T}=\sum_{j=1}^{N_{i}} V_{j i}
$$

$\mathrm{N}_{\mathrm{i}}$ is the number of grains in this class. The rate of change of the total volume of this collection of grains is

$$
\frac{d V_{i}^{T}}{d t}=\frac{d}{d t} \sum_{j=1}^{N_{i}} V_{j i}=\sum_{j=1}^{N_{i}} \frac{d V_{j i}}{d t}=\sum_{j=1}^{N_{i}}-k\left(M_{j i}-\frac{1}{2}\langle\chi\rangle_{j i} L_{j i}\right)
$$


which can be written

$$
\begin{array}{r}
\frac{d V_{i}^{T}}{d t}=-k\left[\sum_{j=1}^{N_{i}} M_{j i}-\frac{1}{2}<\chi>_{i} \sum_{j=1}^{N_{i}} L_{j i}\right] \\
\frac{d V_{i}^{T}}{d t}=-k\left(M_{i}^{T}-\frac{1}{2}<\chi>{ }_{i} L_{i}^{T}\right)
\end{array}
$$

where $\mathrm{M}_{\mathrm{i}}^{\mathrm{T}}$ is the total integral mean curvature of the grains in size class $\mathrm{i}, \mathrm{L}_{\mathrm{i}}{ }^{\mathrm{T}}$ is the length of their edges and $\langle\chi\rangle_{\mathrm{i}}$ is their average dihedral angle.

In order to evaluate the properties on the right side of this equation using the stereological formulae, Eqs. (10) and (11), it is necessary to express them in terms of values per unit volume of structure. The kinetic equation for the grains contained in size class i can be expressed in these terms simply by dividing both sides of the equation by the total volume of the polycrystalline sample, $\mathrm{V}_{0}$ :

$$
\begin{gathered}
\frac{1}{V_{0}} \frac{d V_{i}^{T}}{d t}=-k\left(\frac{M_{i}^{T}}{V_{0}}-\frac{1}{2}<\chi>_{i} \frac{L_{i}^{T}}{V_{0}}\right) \\
\frac{d V_{i}^{T}}{d t}=-k V_{0}\left(M_{V i}-\frac{1}{2}<\chi>_{i} L_{V i}\right)
\end{gathered}
$$

Apply Eqs. (10) and (11) to this set of grains:

$$
\left.\frac{d V_{i}^{T}}{d t}=-k V_{0}\left(2 \pi N_{A i}-\frac{1}{2}<\chi\right\rangle_{i} 2 P_{A i}\right)=-k V_{0}\left(2 \pi N_{A i}-\frac{\pi}{3} P_{A i}\right)
$$


Simplify:

$$
\frac{d V_{i}^{T}}{d t}=-2 \pi k V_{0}\left(N_{A i}-\frac{1}{6} P_{A i}\right)
$$

$V_{i}^{T}$ is the total volume of grains in the size class. Eq. (26) is the working equation used to test the theory.

Measurements of values for $\mathrm{N}_{\mathrm{Ai}}$ and $\mathrm{P}_{\mathrm{Ai}}$ are illustrated in Fig. 1 that shows a two dimensional section obtained from the three dimensional simulation. The grains that are highlighted in this figure are the set of sections obtained from grains in the same three dimensional size class. $\mathrm{N}_{\mathrm{Ai}}$ is a count of those grain sections, per unit area covered by the count, and $\mathrm{P}_{\mathrm{Ai}}$ is a count of their corners (triple points). These counts have been obtained for each size class in the structure. The hypothesis thus predicts that a plot of the rate of change of the total volume of grains in the class versus the quantity in parenthesis on the right side of Eq. (26) is expected to be linear over the range of size classes.

In determining values for these stereological parameters for a given three dimensional size class a total of sixteen contiguous fields on a cross section through the three dimensional simulation structure were examined. The three dimensional size class of each grain section was identified and sections from size class i were highlighted. The number of grain sections in this category was counted, together with the number of triple points in each. Average values were obtained for the sixteen fields observed, then divided by the area of the grain cross section included in the 
observation to give $\mathrm{N}_{\mathrm{Ai}}$ and $\mathrm{P}_{\mathrm{Ai}}$. Standard deviations for each of these statistics were also obtained.

Figure 2 shows a plot that tests Eq. (26) by plotting the left side versus the right side using this simulation data. The data are well described by the least squares line shown in the plot, which also passes through the origin. Confidence intervals of $\pm 1 \mathrm{SE}$ are generally larger at the two ends of the size distribution where the population of grains is smaller. It is concluded that the hypothesis that the evolution of the structure is described by the condition that the local velocity of an element of area is proportional to its local mean curvature is supported by the simulation data.

Average values per grain of $\mathrm{M}, \mathrm{M}_{\mathrm{S}}$, and $\mathrm{M}_{\mathrm{E}}$, were obtained by taking the global values of these parameters for that size class and dividing by the number of grains in the size class. Figure 3 plots these average values versus face class for data obtained from the simulation. Confidence intervals of $\pm 1 \mathrm{SE}$ are again relatively larger for classes with few grains and sections. M, according to MacPherson and Srolovitz, a "natural measure of the length" of a cell, Eq. (9), may be loosely thought of as an adjusted mean caliper diameter [17] of the cell. It is positive over the whole range. The edge contribution to the integral mean curvature, $\mathrm{M}_{\mathrm{E}}$, is also positive over the whole range of faces. The contribution from the cell faces, $\mathrm{M}_{\mathrm{S}}$, which according to Eq. (4) determines the rate of change of the volume of the cell is the difference between these contributions, Eq. (6) and Fig.3. Cells with a small number of faces have convex faces and thus a negative growth rate. For intermediate cell sizes the difference between $\mathrm{M}$ and $\mathrm{M}_{\mathrm{E}}$ passes through zero as does the growth rate. In Fig. 3 this occurs at about $\mathrm{f}=14$. The precise and general condition for zero 
growth rate is $\langle\mathrm{c}\rangle=6$. Cells with a large number of faces have a progressively larger contribution from the saddle edges which results in face geometries that are increasingly concave and contribute negatively to the integral mean curvature. Motion of these negatively curved faces toward their centers of curvature, as required by Eq. (2) leads to the growth of large grains. Thus the contribution of the saddle shaped edges to the integral mean curvature plays a key role in determining the geometries of the cell faces, which controls growth.

\section{Discussion}

This development starts with the classic kinetic assumption that the local velocity of an element of area is proportional to the local mean curvature and leads to the kinetic relation, Eq. (21), with no additional geometric assumptions. It was inspired by von Neumann's result for coarsening of a two dimensional soap froth which begins with the analogous assumption that the local velocity of an element of film boundary is proportional to its local curvature and leads without simplifying geometric assumptions to the equation

$$
\frac{d A_{i}}{d t}=\frac{\pi}{3} k\left(n_{i}-6\right)
$$

where $A_{i}$ is the area of the two dimensional cell and $n_{i}$ is the number of corners or faces on the cell; $\mathrm{k}$ is again the kinetic constant in the original kinetic assumption. In this equation the only geometric factor that determines rate of change of the area of the cell is the number of corners on the cell, a topological parameter. This result is widely referred to as the "n - 6 rule": cells with less than 6 corners shrink while those for which $\mathrm{n}$ is larger than 6 grow. It has long been speculated that an analogous topological rule might govern cell coarsening in three dimensions. 
The development in this paper demonstrates that there is indeed a " $n-6$ rule" that governs coarsening of a three dimensional tessellation under the analogous kinetic assumption. In this case "n" is again the number of corners on a cell, but it is not the topological property of the two dimensions case. It is the stereologically averaged number of corners per cell on all plane sections through the grain. More specifically it is the number of corners on a plane section through the grain averaged over the population of planes that intersect the grain in all positions and orientations. While this number of corners is a topological property of each two dimensional section, its stereological average $\langle\mathrm{c}>$ is not a topological property of the three dimensional polyhedron from which the sections derive, but depends upon its shape and dimensions. In addition it is not the only geometric factor that governs cell evolution, Eq. (21), although it does determine the sign of its rate of change.

Thus the three dimensional result is not a topological version of the two dimensional result. Both developments apply Gibbs thermodynamics of curved surfaces to the kinetic assumption so that the local velocity is proportional to the local mean curvature. In two dimensions the local mean curvature is $1 / r$, where is $r$ is the local radius of curvature of the cell boundary; in three dimensions this property is $\mathrm{H}$, Eq. (3), and contains two parameters. The rate of change of area in two dimensions and volume in three is obtained by integrating the mean curvature, $\mathrm{H}$, over the curved boundaries of the cell. The topological properties are related, through the Gauss-Bonnet theorem [18], to the integral of the local Gaussian curvature over the curved boundaries of the cell; in two dimensions the Gaussian curvature is $1 / \mathrm{r}$; in three dimensions it is the product of the principle curvatures, $\left(1 / r_{1}\right)\left(1 / r_{2}\right)$. Thus in two dimensions the integrals of the mean curvature and the Gaussian curvature are the same, and the integral of the mean curvature gives a topological result, Eq. (27). In three dimensions these two integrals do not give the same result; the integral of 
the Gibbs mean curvature $\mathrm{H}$ is not a topological property in three dimensions.

\section{Summary}

The thermodynamics of curved surfaces suggests the hypothesis that the local velocity of an element of surface area in surface tension driven grain growth is proportional to the local mean curvature. This hypothesis inserted into the kinematic equation relating the change in volume of a grain to the distribution of velocities over its surfaces identifies the integral mean curvature, $\mathrm{M}_{\mathrm{S}}$, of the surfaces on the polyhedral grain as the determining geometric factor in the coarsening process. This parameter is evaluated by applying fundamental stereological equations and yields a new relationship for the rate of change of the volume of the cell that involves the average number of corners on a section through the grain. This result applies to every grain in the structure at every instant in time. It is demonstrated that, while this parameter is not a topological property of the grain, it nonetheless yields a "n - 6 rule" that determines the sign of the growth rate of a grain. This further identifies the condition that separates growing grains from shrinking ones as those for which this average number of corners on a section is 6 .

\footnotetext{
A kinetic Eq. (26) is derived which applies to each size class of grains in the structure and provides the governing equations for its entire growth path envelope. All of the geometric parameters in this equation are experimentally accessible in a serial sectioning experiment providing a very detailed test of the central kinetic hypothesis in normal grain growth. Data from a Monte Carlo simulation of the grain growth process were shown to be consistent with the classic phenomenological hypothesis.
} 


\section{APPENDIX A. Meaning of the Integral Mean Curvature}

The integral mean curvature for a smooth closed surface is defined to be

$$
M=\iint_{S} H d S
$$

where $\mathrm{H}$ is the local value of the mean curvature, Eq. (3) for an element of surface dS. For a convex closed surface Minkowski [19] and Hilliard [20] showed that

$$
M=2 \pi<D>
$$

$\langle\mathrm{D}\rangle$ is the value of the caliper diameter (the distance between parallel tangent planes) averaged over the sphere of normal directions in three dimensions. For closed surfaces in three dimensions that are not completely convex, i.e., that contain saddle and/or concave surface elements, $\langle\mathrm{D}\rangle$ is replaced by MacPherson and Srolovitz' $\mathcal{L}(\mathrm{D})$, Eq. (9), a natural measure of linear size”, which may be loosely thought of as an adjusted mean caliper diameter. In general M remains as defined in Eq. (A1). It is also related to the area feature count $\mathrm{N}_{\mathrm{A}}$ in stereology, Eq. (10), or more generally the Euler characteristic of the set of features on a sectioning plane, footnote 1 . This latter relation makes the measurement of $\mathrm{M}$ possible, but is not much help in trying to visualize the meaning of $\mathrm{M}$.

For polyhedral shapes, i.e. closed surfaces with convex, saddle and concave elements that also have edges, the contribution to $\mathrm{M}$ from the edges may be visualized as follows. For a smooth surface the contribution of an element of surface to the overall value of $\mathrm{M}$ may be defined to be

$$
d M=H d S
$$

This expression can be made more explicit by referring to Fig. A1, which shows an element of area dS with its associated local coordinate system; $\widehat{\mathbb{N}}$ is the normal to the surface and $\widehat{u}$ and $\widehat{\hat{v}}$ are 
axes in the tangent plane defining the local principle normal directions. The two principle radii of curvature, $\mathrm{r}_{1}$ and $\mathrm{r}_{2}$, define the dimensions of $\mathrm{dS}$ by swinging arcs $\mathrm{d} \phi_{1}$ and $\mathrm{d} \phi_{2}$. The dimensions of dS are in the first order, $r_{1} d \phi_{1}$ by $r_{2} d \phi_{2}$. The value of $\mathrm{dM}$ for this element of area is

$$
\begin{aligned}
& d M=H d S=\frac{1}{2}\left(\frac{1}{r_{1}}+\frac{1}{r_{2}}\right) r_{1} d \phi_{1} r_{2} d \phi_{2} \\
& d M=\frac{1}{2}\left(\frac{r_{2}+r_{1}}{r_{1} r_{2}}\right) r_{1} r_{2} d \phi_{1} d \phi_{2} \\
& d M=\frac{1}{2}\left(r_{1}+r_{2}\right) d \phi_{1} d \phi_{2}
\end{aligned}
$$

The contribution of smoothly curved elements of a polyhedral grain to the total value of $\mathrm{M}$ for the body is the integral of this result over the domains of $\phi_{1}$ and $\phi_{2}$ corresponding to the elements of the smoothly curved faces. An element of edge may be thought of as a limiting case in which one of the radii of curvature, say $r_{2}$ goes to zero. For such an element

$$
d M_{E}=\frac{1}{2} r_{1} d \phi_{1} d \phi_{2}
$$

In this expression the product $r_{1} d \phi_{1}$ becomes an element of length, $\mathrm{dL}$, of the edge. Integration over $d \phi_{2}$ gives the local dihedral angle, $\chi$, at the edge; further integration over dL gives

$$
M_{E}=\frac{1}{2} \iint_{\phi_{1 L}} d \phi_{2} d L=\int_{L} \chi d L=<\chi>L
$$

In its general definition the integral mean curvature, $\mathrm{M}$, of a three dimensional closed surface does not have a meaning that can be visualized as do other common general properties like volume and surface area. Nonetheless it arises explicitly in the theory of grain coarsening and fortunately, with the application of results from stereology, leads to results that can be visualized. 


\section{APPENDIX B: Evaluation of $<\mathrm{c}>$ for Flat Faced Polyhedra}

Since $<c>$ is an unfamiliar and abstract property of a polyhedron, this section illustrates its calculation for a number of familiar shapes. They are not intended to be models for grain shapes which typically have curved faces and edges. The computation is straightforward for shapes with flat faces. Recall, Eq. (15), that

$$
<c>=\frac{P_{A}}{N_{A}}=\frac{\frac{1}{2} L_{V}}{\frac{1}{2 \pi} M_{V}}=\pi \frac{L}{M}=\pi \frac{L}{M_{S}+M_{E}}
$$

where $\mathrm{L}$ is the length of edges on the polyhedron, and $\mathrm{M}_{\mathrm{S}}$ and $\mathrm{M}_{\mathrm{E}}$ are the contributions to the integral mean curvature from the faces and edges of the shape for a three dimensional polyhedron with curved faces and edges. For flat faced polyhedra, $\mathrm{M}_{\mathrm{S}}=0$, and

$$
<c\rangle=\pi \frac{L}{M_{E}}=\pi \frac{L}{\frac{1}{2}<x>L}=\frac{2 \pi}{\langle x\rangle}
$$

where $\langle\chi\rangle$ is the average dihedral angle along the edges of the polyhedron,

$$
<\chi>=\frac{\int_{i=1}^{e} X_{i} L_{\bar{i}}}{\sum_{i=1}^{e} L_{i}}
$$

Values for $\langle\mathrm{c}>$ for some regular polyhedra (in the sense that all edges are the same length) were computed using vector analysis and are shown in Table 1 in the text. 


\section{Acknowledgement}

The authors gratefully acknowledge financial support from NSF Grant No. DMR-1307665.

\section{References}

[1] J. von Neumann, written discussion in: Metal Interfaces, American Society for Metals, Cleveland, 1952, pp.108-110.

[2] C.S. Smith, Grain shapes and other metallurgical applications of topology, in: Metal Interfaces, American Society for Metals, Cleveland, 1952, pp.65-108.

[3] R.T. DeHoff, Thermodynamics in Materials Science, second ed., Taylor and Francis, Boca Raton, 2006, Ch.12, Capillarity effects in thermodynamics.

[4] R.D. MacPherson, D.J. Srolovitz, The von Neumann relationship generalized to coarsening of three-dimensional microstructures, Nature 446 (2007) 1053-1055.

[5] W.W. Mullins, Two-Dimensional Motion of Idealized Grain Boundaries, J Appl Phys 27 (1956) 900-904.

[6] D. Harker and E. R. Parker, Grain shape and grain growth, Trans. ASM 34 (1945) 156-195.

[7] C.S. Smith, Grains, phases, and interfaces-an interpretation of microstructure, Trans. Met. Soc. AIME 175 (1948) 15-51.

[8] J.W. Cahn, W.C. Hagel, Divergent pearlite in a manganese eutectoid steel, Acta Metall 11 (1963) 561-574. 
[9] R.T. DeHoff, The geometrical meaning of the integral mean curvature, in: Microstructural

Science, J.D. Braun, H.W. Arrowsmith, J.L. McCall eds., Elsevier, New York, 1977, pp.331-348.

[10] R.T. DeHoff, G.Q. Liu, On the relation between grain size and grain topology, Metall Trans A 16A (1985) 2007-2011.

[11] R.T. DeHoff, F.N. Rhines (Eds.), Quantitative Microscopy McGraw-Hill, New York, 1968.

[12] R.T. DeHoff, The quantitative estimation of mean surface curvature, Trans. Met. Soc. AIME, 239 (1967) 617-621.

[13] J.W. Cahn, The significance of average mean curvature and its determination by quantitative metallography, Trans. Met. Soc. AIME 239 (1967) 610-616.

[14] Ibid. 11, p.87, pp. 291-324.

[15] C.V. Howard, M.G. Reed, Unbiased Stereology, second ed., Garland Science/Bios, New York, 2005.

[16] W. Thomson, On the division of space with minimum partitional area, Phil. Mag. 24 (1887) 503-514.

[17] Ibid. 11, pp. 131-133.

[18] D.J. Struik, Lectures on Classical Differential Geometry, Addison Wesley, Cambridge, 1950, pp.153-159.

[19] H. Minkowski, Volumen und Oberfläche, Math. Ann. 57 (1903) 447-495. 
[20] J. E. Hilliard, The Calculation of the Mean Caliper Diameter of a Body for Use in the Analysis of the Number of Particles Per Unit Volume, in: Stereology, Springer, Berlin Heidelberg, 1967, pp. 211-215. 
Figure captions

Figure 1. Highlighted grains belong to the same three dimensional size class. $N_{A}$ is a count of those grain sections; $P_{A}$ is a count of their number of corners.

Figure 2. Test of the hypothesis that the local velocity of an element of area in the simulation is proportional to its mean curvature.

Figure 3. Average values of $\mathrm{M}, \mathrm{M}_{\mathrm{S}}$, and $\mathrm{M}_{\mathrm{E}}$ (integral mean curvatures for the polyhedron, its faces and edges) for grains in each size class are plotted versus the average number of faces for those grains.

Figure A1. Illustration of an element of area of a curved surface showing the local coordinate system, principle radii of curvature, $\mathrm{r}_{1}$ and $\mathrm{r}_{2}$, and the angles subtended. 
Figure 1. Highlighted grains belong to the same three dimensional size class. $N_{A}$ is a count of those grain sections; $P_{A}$ is a count of their number of corners.

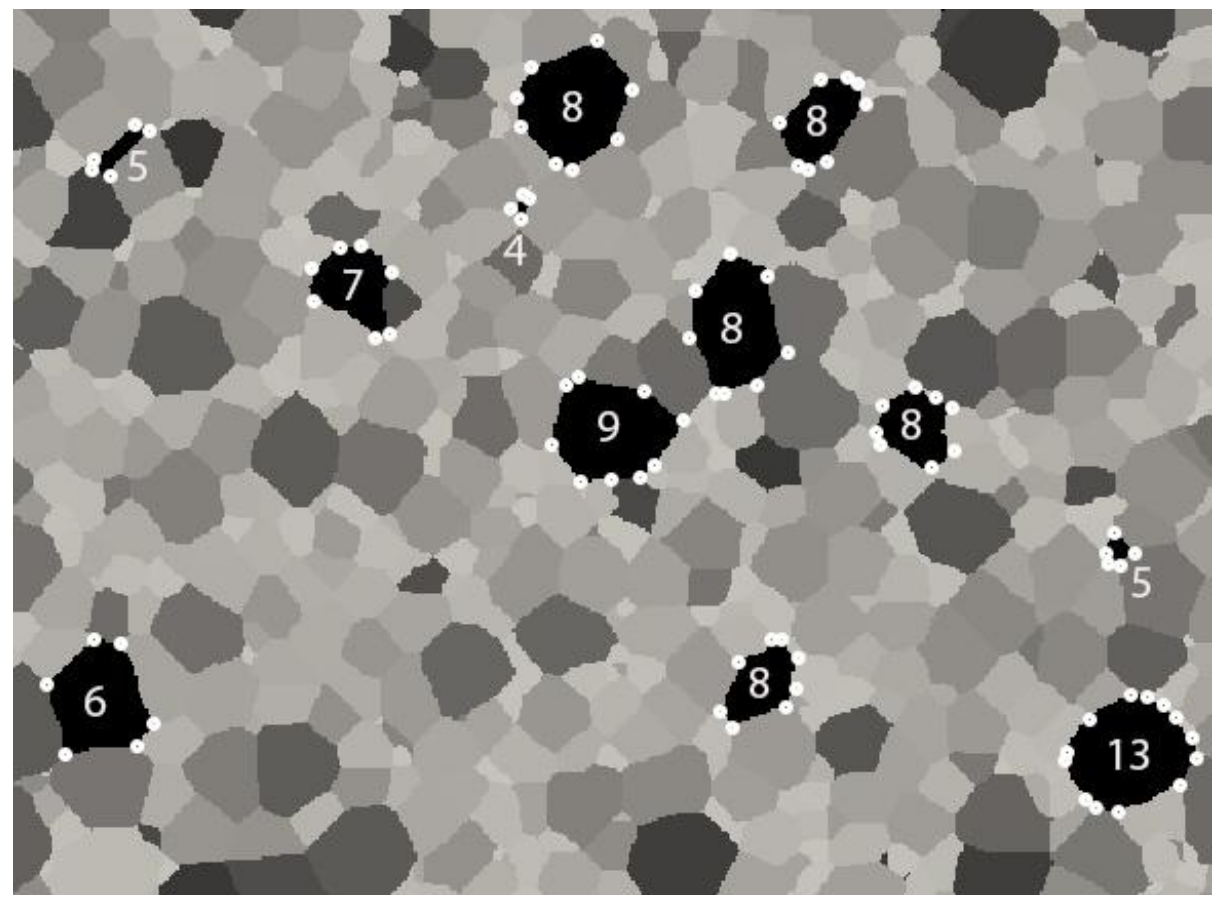


Figure 2. Test of the hypothesis that the local velocity of an element of area in the simulation is proportional to its mean curvature.

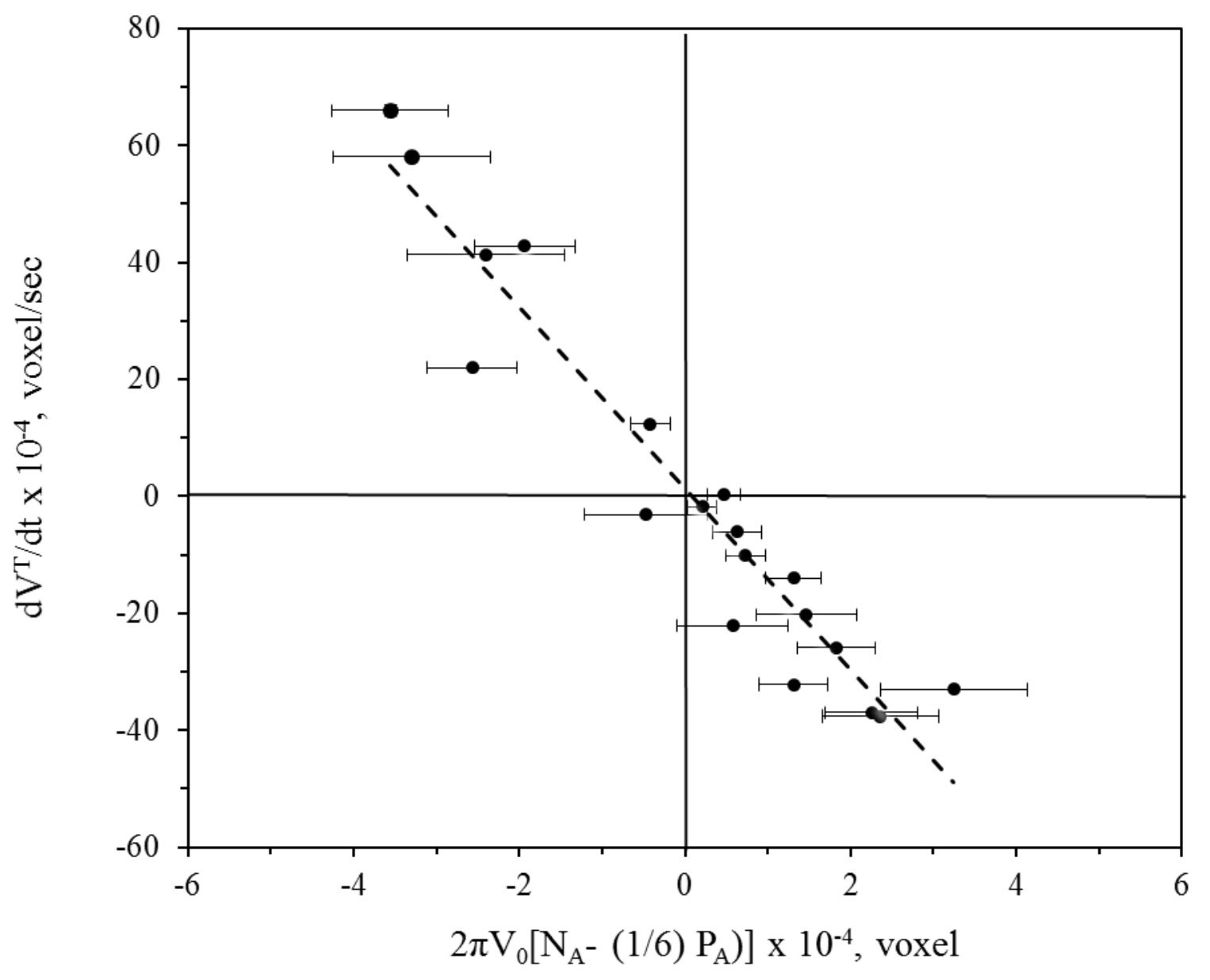


Figure 3. Average values of $\mathrm{M}, \mathrm{M}_{\mathrm{S}}$, and $\mathrm{M}_{\mathrm{E}}$ (integral mean curvatures for the polyhedron, its faces and edges) for grains in each size class are plotted versus the average number of faces for those grains.

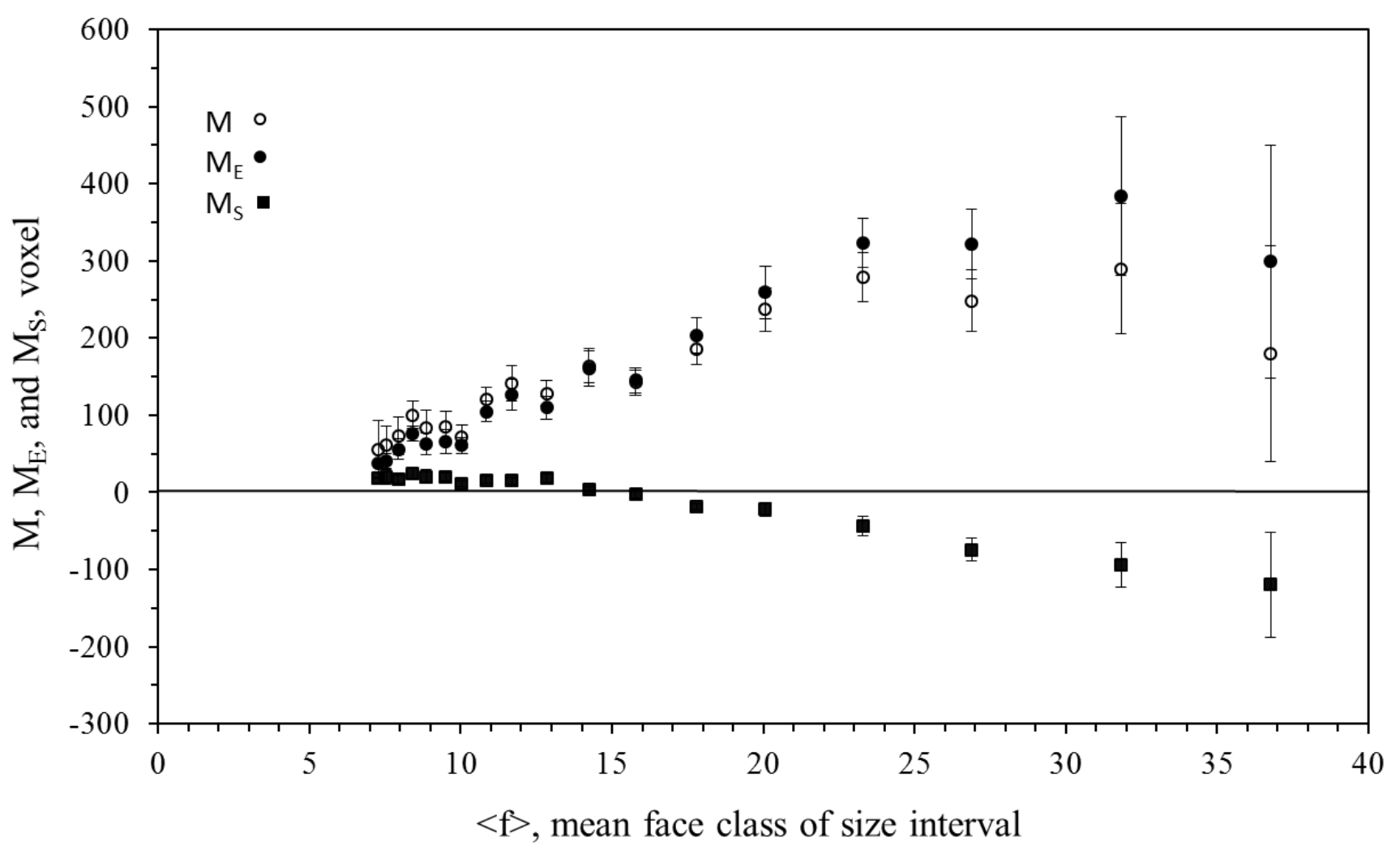


Figure A1. Illustration of an element of area of a curved surface showing the local coordinate system, principle radii of curvature, $r_{1}$ and $r_{2}$, and the angles subtended.

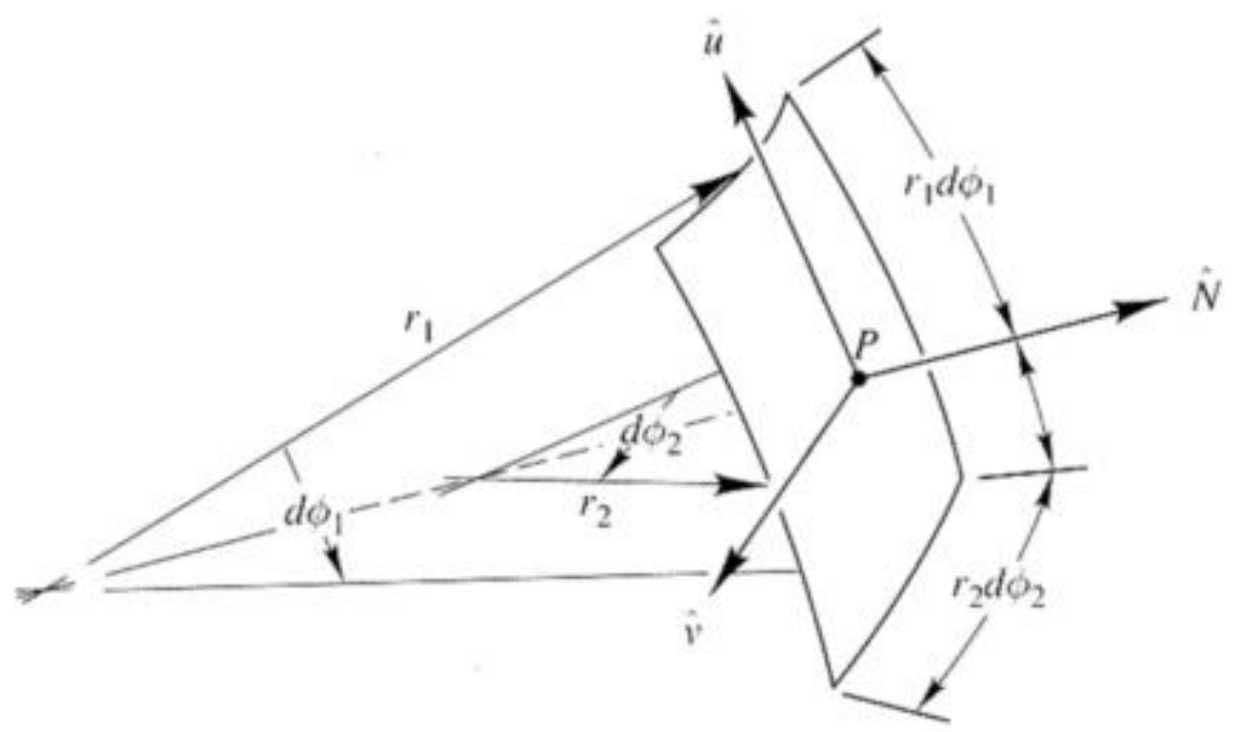


Table 1. Values of $<c>$ for selected regular polyhedra.

\begin{tabular}{|c|c|c|c|}
\hline Polyhedron & Faces & $<\chi>$ & $\langle c\rangle$ \\
\hline Tetrahedron & 4 & $\cos ^{-1}(-1 / 3)=1.91063$ & 3.288 \\
\hline Triangular Prism & 5 & {$[6(\pi / 2)+2(2 \pi / 3)] / 9=(5 / 9) \pi$} & 3.600 \\
\hline Cube & 6 & $\pi / 2$ & 4.000 \\
\hline Octahedron & 8 & $\cos ^{-1}(1 / 3)=1.2309$ & 5.104 \\
\hline Tetrakaidecahedron & 14 & {$\left[24 \cos ^{-1}(1 / \sqrt{3})+12 \cos ^{-1}(1 / 3)\right] / 36=\pi / 3$} & 6.000 \\
\hline
\end{tabular}



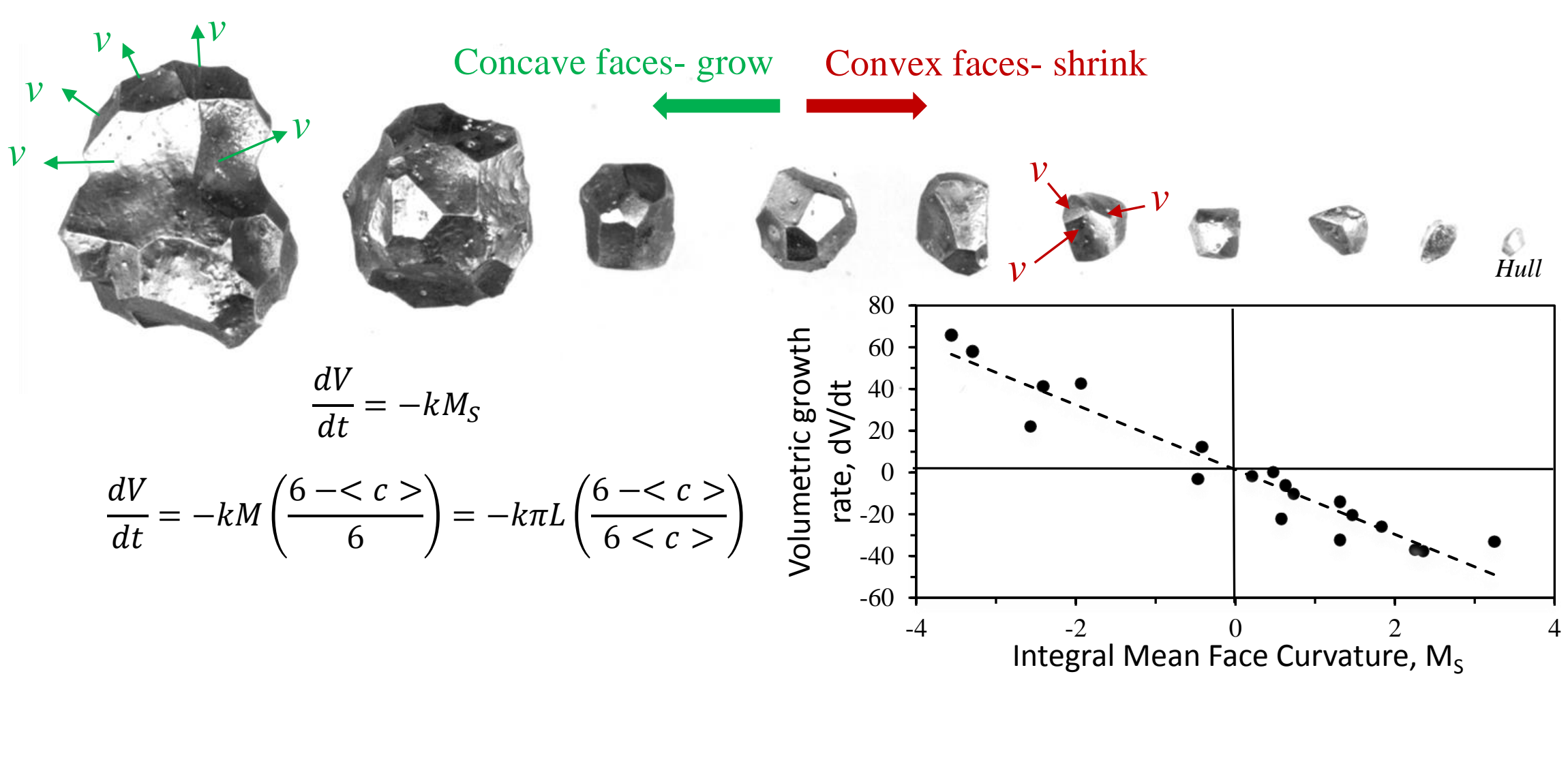

$$
\text { ( }
$$

(n)$$
\frac{d V}{d t}=-k M\left(\frac{6-<c>}{6}\right)=-k \pi L\left(\frac{6-<c>}{6<c>}\right)
$$
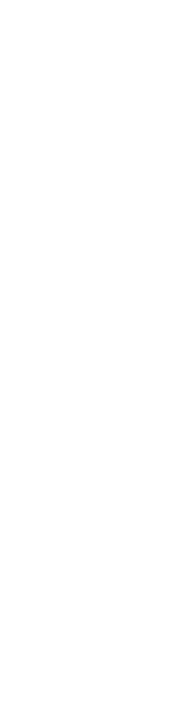